\title{
Primary Isolated Gingival Tuberculosis
}

\author{
Gabriel Rodrigues ${ }^{1}$, Sunitha Carnelio ${ }^{2}$ and Maanna Valliathan ${ }^{3}$ \\ ${ }^{1}$ Departments of Surgery, ${ }^{2}$ Oral Pathology, ${ }^{3}$ General Pathology, Kasturba Medical College; Manipal, Karnataka, India
}

\begin{abstract}
Tuberculosis of the oral cavity is a rare event and when present is usually secondary to pulmonary tuberculosis. We present a case of a 33-year-old male patient who presented with symptoms of gingivitis. Local examination revealed multiple ulcers and a few nodules over the gingiva. A nodule biopsy was consistent with the diagnosis of gingival tuberculosis. The patient had no evidence of tuberculosis any where else in the body. Medical treatment was curative. As this condition is very rare, it is often over looked as it is never thought as a common clinical diagnosis. A biopsy is mandatory to establish diagnosis. The relevant literature is reviewed.

Key-Words: Gingiva, tuberculosis, mycobacteria, antituberculous treatment.
\end{abstract}

Since the introduction of effective chemotherapy, tuberculous lesions of the oral cavity have become so infrequent that it is virtually a forgotten disease entity and pose a diagnostic problem. They account for less than one percent of cases of extrapulmonary tuberculosis, are usually associated with foci of disease elsewhere in the body and enlarged, palpable cervical lymph nodes are usually present [1]. Primary oral tuberculous lesions are much rarer, as early diagnosis and treatment of tuberculosis (TB) elsewhere in the body may be the reason for its uncommon presentation. Tongue ulcer is the commonest form of presentation of oral TB, followed by gingival involvement [2]. Its infrequent clinical presentation and increased chance of being overlooked during routine intraoral examination make it worthy of documentation. A review of literature of the past 55 years revealed only three cases of primary isolated gingival TB. Although the incidence of disease is continuously decreasing, TB still remains a major health problem, especially in India. The purpose of this article is to emphasize the importance of early diagnosis of primary TB of oral cavity and especially of gingiva, which may be misdiagnosed when oral lesions are not associated with any apparent systemic infection.

\section{Case Report}

A 33-year-old male presented with complaints of reddening and painful gingival ulceration, mild intermittent fever and lethargy of two months duration. There was no history of dental trauma, cough with expectoration, fever or weight loss and his appetite was normal. On examination, his general condition and vital signs were normal. There was no cervical lymphadenopathy. Systemic review was unremarkable. Local examination revealed red, tender and multiple ulcerated lesions involving the labial vestibule from left premolar to the opposite counterpart in the upper jaw. A few nodules were also present on the gingiva. The teeth and rest of the oral cavity was normal with good oral hygiene.

Received on 19 July 2006; revised 18 December 2006.

Address for correspondence: Dr. Gabriel Rodrigues. 157, KMC Quarters Madhav Nagar, Manipal - 576 104. Karnataka, India. Phone: +91-8202572647. Fax: +91-820-2570061. E-mail: rodricksgaby@yahoo.co.in.

The Brazilian Journal of Infectious Diseases 2007;11(1):172-173. (C) 2007 by The Brazilian Journal of Infectious Diseases and Contexto Publishing. All rights reserved.
Investigations revealed a hemoglobin of $12 \mathrm{gm} / \mathrm{dL}$, WBC count of $5.3 \times 10^{9} / \mathrm{L}$, polymorphs 40 percent, lymphocytes 20 percent, and the ESR was $10 \mathrm{~mm} / \mathrm{h}$. Serological studies (ELISA for HIV and VDRL) were non-reactive. A PPD test was performed but was found to be negative. A chest X-ray (CXR) was normal. A provisional differential diagnosis of vitamin B12 deficiency, tuberculosis, Wegener's granulomatosis, sarcoidosis and malignancy was made and biopsy of a nodule was performed. Histopathological examination showed an atrophic ulcerated epithelium, caseating granulomas surrounded by lymphocytes, epitheloid cells and Langhans giant cells (Figure 1) and thus a diagnosis of primary isolated gingival tuberculosis was made. The patient was put on antituberculous treatment (isoniazid, rifampicin, pyrazinamide, and ethambutol). On completion of six months of chemotherapy there has been a complete resolution of the disease and during follow-up there has been no recurrence.

\section{Discussion}

TB is a chronic infectious granulomatous disease and is very common in India and Southeast Asia, where the prevalence rate is about four every 1,000 people and the incidence rate of the disease is of two percent. Fifteen percent of the tuberculous population of the world resides in India [2].

Gingival TB is caused by Mycobacterium tuberculosis and less frequently by Mycobacterium bovis, both being acid-

Figure 1. Photomicrograph showing epitheloid granulomas, caeseation and Langhans giant cells 40x (HE).

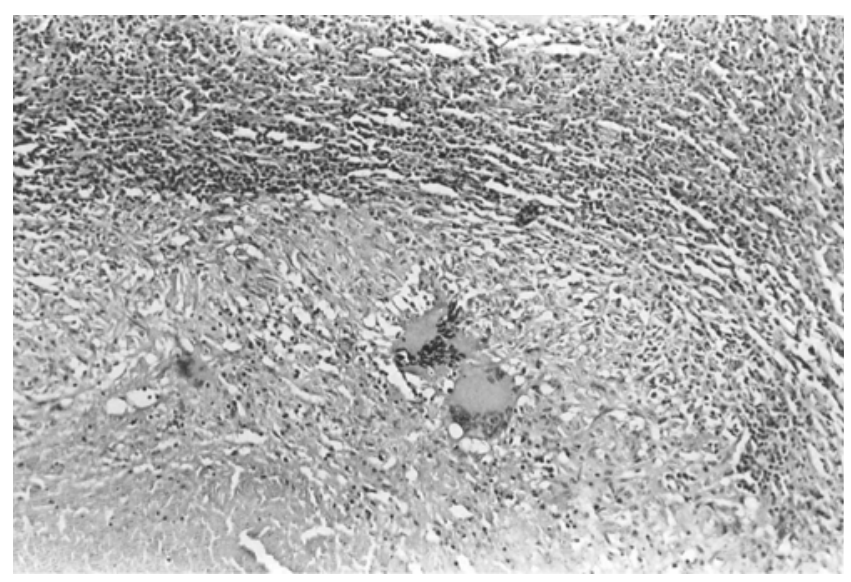


fast bacilli (AFB) and is usually associated with TB of the oropharynx, lungs, lymph nodes and military TB. Gingival TB (primary and secondary) has been reported but all have been associated either with candidiasis, HIV infection, pulmonary TB or cervical lymphadenopathy [3] . Primary isolated gingival TB is extremely rare and we could find only three cases documented in the literature [4].

The tongue and gingiva are the most common sites of infection in patients with oral TB, followed by that of tooth sockets, soft palate, floor of mouth, lips and buccal mucosa. Primary gingival involvement is more common in children and adolescents than adults. It usually presents as a single painless indolent ulcer, which progressively extends from the gingival margin to the depths of the adjacent vestibule and is often associated with enlarged cervical lymph nodes [2]. Oral lesions can also present as nodules, fissures, plaques, vesicles, tuberculomas or granulomas and the last two are usually caused by underlying pulmonary disease. They may be single or multiple, painful or painless and usually appear as irregular, well circumscribed ulcer with surrounding erythema without induration and satellite lesions are commonly found [1].

The pathogenesis is usually self inoculation with infected sputum, but hematogenous spread also occurs. Direct inoculation commonly involves the gingiva, dental sockets and buccal folds. It is believed that an intact squamous epithelium of the oral mucosa is relatively resistant to penetration of tuberculous bacilli and that saliva has some inhibitory effect on mycobacteria. Small mucosal tears may favor the localization of organisms. Other predisposing factors include poor oral hygiene, dental extraction and leucoplakia [5]. Gingival TB should be differentiated from traumatic ulcers, aphthous ulcers, actinomycosis, candidiasis, syphilitic ulcers, Wegeners granulomatosis, sarcoidosis and malignancies including lymphoma. A biopsy, as in our patient, is necessary to confirm the diagnosis. A CXR and a Mantoux skin test are mandatory to rule out systemic TB. The treatment of oral TB is by anti tuberculous drugs.

With the decreased incidence of TB, unusual manifestations of oral TB are likely to be missed. The clinician needs to be aware of these various lesions and should consider TB in the differential diagnosis of any nodular, ulcerated lesions of the oral cavity.

\section{References}

1. Eng H.L., Lu S.Y., Yang C.H., Chen W.J. Oral tuberculosis. Oral Surg Oral Med Oral Pathol Oral Radiol Endod 1996;81:415-20.

2. Carnelio S., Rodrigues G. Primary lingual tuberculosis: a case report with review of literature. J Oral Sci 2002;44:55-7.

3. Dimitrakopoulos I., Zouloumis L., Lazaridis N., et al. Primary tuberculosis of the oral cavity. Oral Surg Oral Med Oral Pathol Oral Radiol Endod. 1991;72:712-5.

4. Srivastava A., Chacko R., John L. Isolated gingival tuberculosis. Indian J Chest Dis Allied Sci 1986;28:166-8.

5. Gupta A., Shinde K.J., Bhardwaj I. Primary lingual tuberculosis: a case report. J Laryngol Otol 1998;112:86-7. 